\title{
Photoabsorption of hydrocarbons in Titan's atmosphere
}

\author{
Fabíola P. Magalhães, Gerardo G. B. de Souza \\ and Heloisa M. Boechat-Roberty
}

Universidade Federal do Rio de Janeiro - Observatório do Valongo, Brazil email: fabiola02@astro.ufrj.br

\begin{abstract}
Titan, the largest satellite of the planet Saturn, has a thick atmosphere which consists of nitrogen $\left(\mathrm{N}_{2}\right)$ and methane $\left(\mathrm{CH}_{4}\right)$. In 2004, the Cassini-Huygens mission observed the occultation of two stars through the atmosphere of Titan and measured ultraviolet (UV) absorption spectra. Through these spectra it was possible to identify the molecular species contained in this environment. In the present work, we have simulated a spectrum of this atmosphere using some molecules such as $\mathrm{CH}_{4}, \mathrm{C}_{2} \mathrm{H}_{2}, \mathrm{C}_{2} \mathrm{H}_{4}, \mathrm{C}_{2} \mathrm{H}_{6}, \mathrm{C}_{4} \mathrm{H}_{2}$, and $\mathrm{C}_{6} \mathrm{H}_{6}$. Our cross sections data were experimentally obtained using the electron energy-loss technique, where the electron energy-loss spectra, measured high incident energies and in small scattering angles, are similar to photoabsorption spectra. The comparison of our synthetic spectrum with that measured by Cassini shows that this method is very efficient for identifying molecules as well as estimating abundances.
\end{abstract}

\section{Introduction}

Titan's atmosphere is primarily composed of nitrogen but methane is the nextmost abundant constituent (Wilson \& Atreya 2004). The interaction of solar X-rays and ultraviolet photons or energetic electrons with these constituents produce a rich and complicated organic chemistry that leads to the formation of new molecules such as hydrocarbons and nitriles. These latter are organic species containing a triple CN bond, such as HCN, which is a precursor of the nitrogenous base of DNA, the adenine molecule. In 2004, the Cassini-Huygens mission observed the occultation of two stars through the atmosphere of Titan, Shaula ( $\lambda$ Scorpion) and Spica ( $\alpha$ Virgo) and obtained UV absorption spectra from $110 \mathrm{~nm}$ to $190 \mathrm{~nm}$. This atmospheric transmission of the stellar flux can be understood by the Lambert-Beer law:

$$
I(\lambda)=I_{0}(\lambda) e^{-\tau} \longrightarrow \ln \left(\frac{I}{I_{0}}\right)=-\tau(\lambda)
$$

where $I(\lambda)$ is the stellar flux as a function of the wavelength $(\lambda)$ after being attenuated by the absorbing material in Titan's atmosphere, $\mathrm{I}_{0}$ is the stellar flux before traversing the atmosphere and $\tau(\lambda)$ is the optical depth given by:

$$
\tau(\lambda)=\Sigma_{i} \sigma_{i}(\lambda) N_{i}
$$

where $\sigma_{i}(\lambda)$ is the cross section $\left(\mathrm{cm}^{2}\right)$ and $N_{i}$ is the column density $\left(\mathrm{cm}^{-2}\right)$ for each molecular species $i$.

\section{Methodology}

We have obtained photoabsorption spectra converted from the electron energy-loss spectra for molecules such as benzene, naphthalene and anthracene (Boechat-Roberty 
et al. 2004, de Souza et al. 2002, Boechat-Roberty et al. 1997) and other species. Briefly, we have measured electron energy-loss spectra (EELS) at electron incident energy of $1000 \mathrm{eV}$ and small scattering angles. In these conditions, the generalized oscillator strength (measured by EELS) tends to be the optical oscillator strength (Lassettre \& Skerbele 1974). To obtain the photoabsorption cross-section, each point in the EELS was extrapolated to zero scattering angle using the universal formula (Boechat-Roberty et al. 2004). To compute the Titan synthetic spectrum, we used the cross-sections of the following species: $\mathrm{CH}_{4}, \mathrm{C}_{2} \mathrm{H}_{2}, \mathrm{C}_{2} \mathrm{H}_{4}, \mathrm{C}_{2} \mathrm{H}_{6}, \mathrm{C}_{4} \mathrm{H}_{2}$, and $\mathrm{C}_{6} \mathrm{H}_{6}$. The column densities, initially adopted from Shemansky et al. (2005), were adjusted to achieve the best fit.

\section{Results and conclusions}

Figure 1 represents the synthetic spectrum obtained from the cumulative product of the photoabsorption cross-section by the respective column density of each molecule. The comparison of our result with the Cassini spectrum shows a very good agreement.

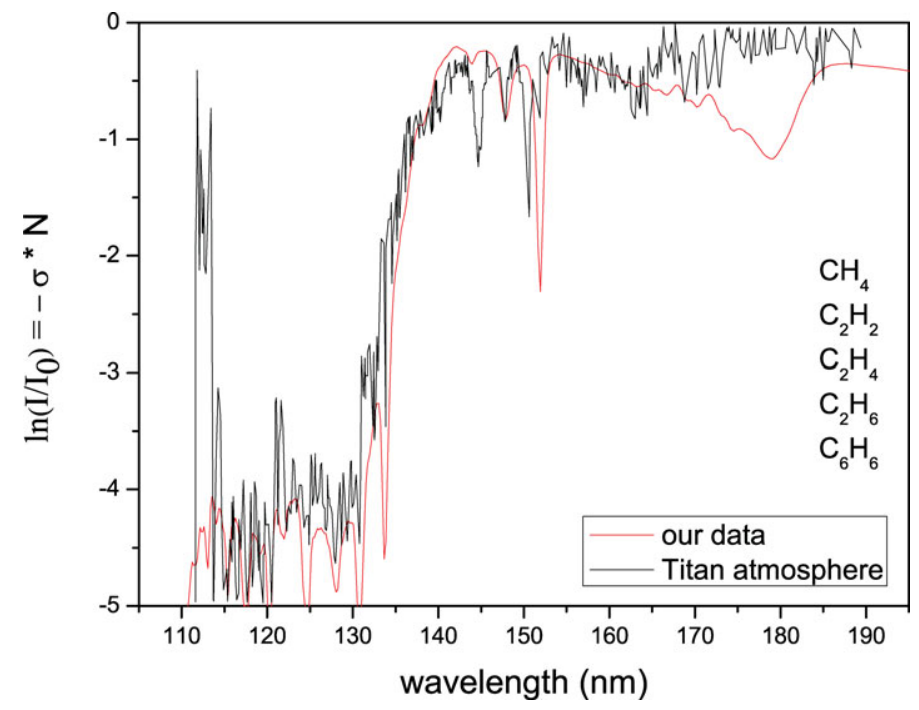

Figure 1. Comparison of our synthetic spectrum with the spectrum obtained by the Cassini spectrometer.

\section{Acknowledgements}

The authors would like to thank the financial support from Conselho Nacional de Desenvolvimento Cientfico e Tecnolgico (CNPq) and FAPERJ.

\section{References}

Boechat-Roberty, H. M., Rocco, M. L. M., Lucas, C. A., Fernandes, M. B., \& de Souza, G. G. B. 1997, J. Phys. B: At. Mol. Opt. Phys. 30, 3369

Boechat-Roberty H. M., Rocco, M. L. M., Lucas, C. A., \& de Souza, G. G. B. 2004, J. Phys. B: At. Mol. Opt. Phys. 37, 1467

de Souza, G. G. B., Boechat-Roberty, H. M., Rocco, M. L. M., \& Lucas, C. A. 2002, J. Electron Spec \& Rel Phen. 123, 315

Lassetre, E. N. \& Skerbele, A. 1974, Methods Exp. Phys. B3, 868

Shemansky, D. E., Stewart, A. I. F., West, R. A., Esposito, L. W., Hallett, J. T., \& Liu, X. 2005, Science 308, 978

Wilson, E. H. \& Attreya, S. K. 2004, J. Geophys. 109, E06002 